\title{
Neurophotonics: non-invasive optical techniques for monitoring brain functions
}

\author{
Alessandro Torricelli, PhD \\ Davide Contini, PhDa \\ Alberto Dalla Mora, PhDa \\ Antonio Pifferi, PhD ${ }^{a}$ \\ Rebecca Re, PhDa \\ Lucia Zucchelli, PhDa \\ Matteo Caffini, $\mathrm{PhD}^{\mathrm{b}}$ \\ Andrea Farina, $\mathrm{PhD}^{\mathrm{c}}$ \\ Lorenzo Spinelli, PhD'
}

\author{
aDipartimento di Fisica, Politecnico di Milano, Milan, \\ Italy

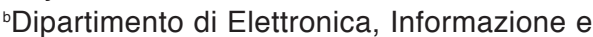 \\ Bioingegneria, Politecnico di Milano, Milan, Italy \\ 'Istituto di Fotonica e Nanotecnologie, Consiglio \\ Nazionale per le Ricerche, Milan, Italy
}

Correspondence to: Alessandro Torricelli

E-mail: alessandro.torricelli@polimi.it

\section{Summary}

The aim of this review is to present the state of the art of neurophotonics, a recently founded discipline lying at the interface between optics and neuroscience. While neurophotonics also includes invasive techniques for animal studies, in this review we focus only on the non-invasive methods that use near infrared light to probe functional activity in the brain, namely the fast optical signal, diffuse correlation spectroscopy, and functional near infrared spectroscopy methods. We also present an overview of the physical principles of light propagation in biological tissues, and of the main physiological sources of signal. Finally, we discuss the open issues in models, instrumentation, data analysis and clinical approaches.

KEY WORDS: diffuse correlation spectroscopy, fast optical signal, functional near infrared spectroscopy, neurophotonics

\section{Introduction}

Photonic technologies have, in recent years, been playing an increasingly big role in supporting the study of living organisms (e.g. cells, small animal and human) in biology and medicine. Biomedical applications like spectroscopy and imaging of biological tissues are now a major field of application for light-based technologies, and the term biophotonics is commonly used (Popp et al., 2012). More specifically, the methods and applications based on the use of light in neuroscience to further understanding of brain phenomena are growing rapidly and a new term - neurophotonics - has recently been coined to refer to all the disciplines that are working actively and in synergy at the interface between optics and neuroscience. These methods and applications embrace a cornucopia of solutions and tools, such as microscopic and super-resolution nanoscopic methods (Nieuwenhuizen et al., 2013), optogenetics and other optical methods of manipulating cellular behavior (Welberg, 2013), the use of synthetic and genetically encoded optical reporters and actuators (Siegel and Isacoff, 1997; Guerrero et al., 2002; Ataka et al., 2002), optical clearing methods (Zhu et al., 2013), optical methods for investigating neuroglial and vascular physiology, optical methods for investigating cellular energetics (Devor and Boas, 2012), and noninvasive optical methods for imaging brain function (Hillman, 2007; Obrig and Villringer, 2003; Ferrari and Quaresima, 2012). This field has become so important that 2014 saw the launch of a new journal "Neurophotonics" devoted to it (www.spie.org).

In this review, we present the non-invasive optical methods of imaging brain function in humans. The interest in using optical methods to non-invasively probe the brain is due to the fact that cerebral activity influences the optical properties of brain tissue. A detailed description of the physiological processes associated with brain activity can be found in Villringer and Chance (1997). In figure 1, we summarize the main physiological sources of contrast for optical parameters and link the measured optical parameters to the techniques effectively used to indirectly measure the underlying brain activity.

The first source of contrast is related to the cellular physiological events associated with brain activity. Ion and water fluxes across neuronal membranes affect the membrane potential and can influence light scattering. Brain activity-related light scattering changes have been measured in vitro (Hill and Keynes, 1949; Stepnoski et al., 1991; Lipton, 1973; MacVicar and Hochman, 1991; Salzberg and Obaid, 1988) and in vivo, both in intact animals (Malonek and Grinvald, 
1996) and in human adults (Gratton et al., 1995) by means of the fast optical signal (FOS) technique. Other optical techniques exploit the so-called neurovascular coupling mechanism, the underlying basis of the blood oxygen level dependent (BOLD) signal. The BOLD signal is commonly monitored by functional magnetic resonance imaging (fMRI) (Ogawa, et al., 1992), but regional blood flow changes following a neuronal activation can also be detected, thanks to the different absorption spectra of oxygenated and deoxygenated hemoglobin $\left(\mathrm{HbO}_{2}\right.$ and $\mathrm{Hb}$, respectively) in the near infrared (NIR) window (Villringer and Chance, 1997; Obrig and Villringer, 2003), by functional near infrared spectroscopy (fNIRS) or, thanks to the Doppler shifts induced by the motion of red blood cells, by diffuse correlation spectroscopy (DCS).

We here focus on these three major techniques: FOS, DCS and fNIRS. The principles, instrumentation, and applications of each technique will be briefly reviewed. To properly understand the basics of these methods, a brief overview of the physical principles of light propagation in biological tissues, and of the main physiological sources of signal, is provided. Finally, the open issues in models, instrumentation, data analysis and clinical approaches are discussed.

\section{Photon migration in biological media}

Electromagnetic radiation in the visible $(400-700 \mathrm{~nm}$, VIS) and near infrared (700-2000 nm, NIR) spectral region is generally referred to as light (Born and Wolf, 1999). Like many materials (e.g. powders, paint, clouds), biological tissues are opaque to VIS and NIR light due to the phenomena of light absorption and light scattering. The origin of these phenomena lies in the spatial and frequency changes in the dielectric properties occurring at the microscopic level (Bohren and Huffman, 1983). In biological tissues the presence of chromophores like hemoglobin, water, lipids and collagen contributes to light absorption, while the shape and size of cells and intracellular structures (e.g. nuclei, mitochondria) contribute to light scattering (Dunn and Richards-Kortum, 1996; Mourant et al., 1998). The overall light distribution in a biological tissue is determined by the interplay between light absorption and light scattering. In the VIS and NIR spectral range light scattering can assume values in the range of 5-30 $\mathrm{cm}^{-1}$ which are generally much larger than those of light absorption, which generally remain within the range of $0.02-2 \mathrm{~cm}^{-1}$ (Jacques, 2013). For this reason, biological tissues are usually referred to as diffusive media. While the high absorption of hemoglobin at shorter wavelengths $(<600 \mathrm{~nm})$ and of water at longer wavelengths $(>1100 \mathrm{~nm})$ limits light penetration in the tissue to few millimeters, in the so-called therapeutic and diagnostic window (between 650 and $850 \mathrm{~nm}$ ) light absorption is commonly very low $\left(<0.2 \mathrm{~cm}^{-1}\right)$ with the result that light penetrates to a depth of a few centimeters (Fig. 2). This makes it possible to non-invasively probe biological tissue in depth and allows interesting biomedical applications like optical mammography (Taroni et al., 2012) and muscle and brain oximetry (Contini et al., 2012).

The terms diffuse optical spectroscopy (DOS) and diffuse optical imaging (DOI) indicate the methodologies that use VIS and NIR light to non-invasively probe diffusive media like biological tissues. Typically, in a DOS measurement, light is delivered to and collected from the sample by means of optical fibers (optodes), or by placing light sources and detectors directly in contact with the probed tissue. The simplest DOS measurement configuration is the transmittance mode in which the injection and collection fibers are positioned on opposite surfaces. In the biomedical field this is possible only for a few applications such as hemorrhage detection in newborns (Gibson et al., 2006), thanks to the small size and transparency of the newborn's head, optical mammography, where the female breast is gently compressed by parallel transparent plates (Taroni et al., 2012), or finger arthritis detection (Golovko et al., 2011), where it is possible

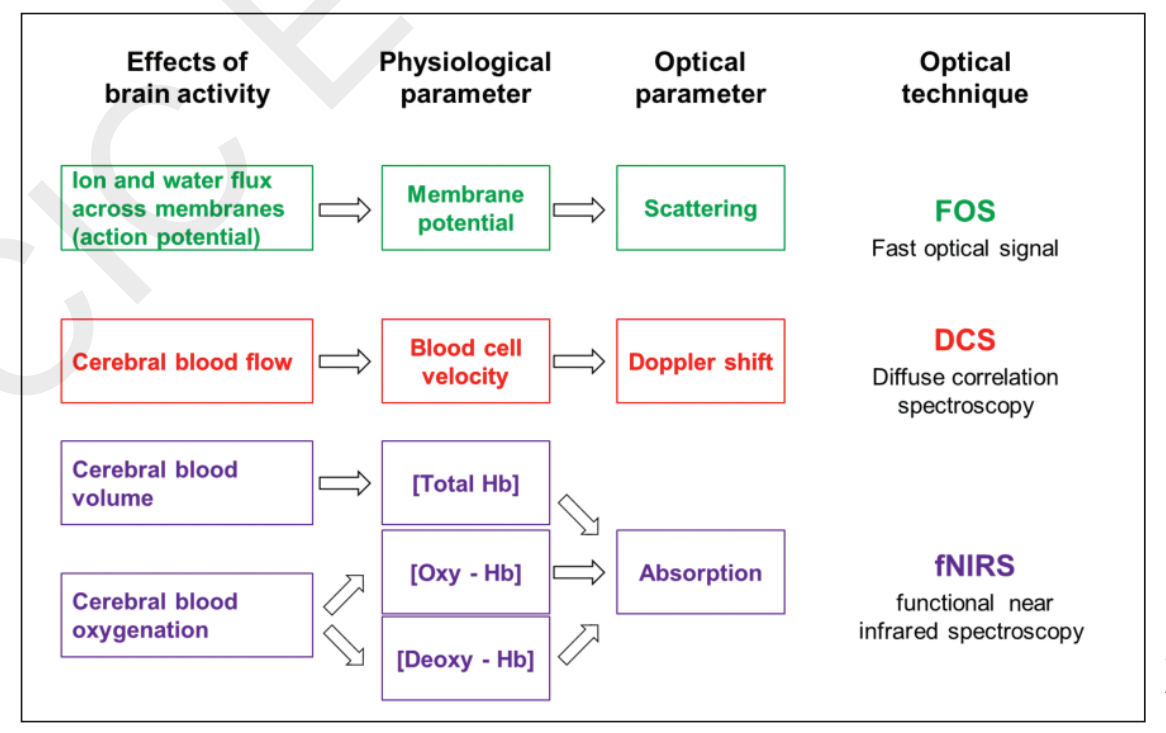

Figure 1 - Assessment of brain activity by optical methods. Adapted from Villringer and Chance (1997) 
thanks to the thin shape of the finger. On the other hand, the reflectance mode exploits the fact that, thanks to scattering, light is highly diffused in the sample volume and DOS measurements become possible by placing a couple of optic fibers on the same surface of the tissue at a distance of a few centimeters. For DOI a combination of several injection and collection fibers positioned in regularly spaced arrangements allows topographic or tomographic approaches (Arridge, 1999).

Independently of the measurement geometry, three different DOS/DOI approaches can be implemented: i) continuous wave $(\mathrm{CW}) \mathrm{DOS} / \mathrm{DOI}$ makes use of a steady-state light source (e.g. a light-emitting diode or a laser with a constant intensity over time) that can typically be amplitude modulated at a low (a few $\mathrm{kHz}$ ) frequency in order to exploit the significant noise reduction available with phase-locked detection techniques, and a detection apparatus sensitive to light attenuation changes (e.g. photodiode);

ii) frequency domain (FD) DOS/DOI is based on amplitude-modulated light sources (at frequencies of the order of $100 \mathrm{MHz}$ or larger, up to $\sim 1 \mathrm{GHz}$ ) and on the detection of light amplitude demodulation and phase shift;

iii) time domain (TD) DOS/DOI employs a pulsed light source, typically a laser providing light pulses with a duration of a few tens of picoseconds, and a detection apparatus with temporal resolution in the subnanosecond scale. A detailed review of these different approaches can be found in Durduran et al. (2010).

As shown in Figure 3, in the reflectance configuration the volume that is, on average, probed by CW DOS is the so-called banana shape region, which reaches a depth roughly equivalent to half the source-detector distance (Del Bianco et al., 2002). To reach the adult brain cortex, a distance greater than $3 \mathrm{~cm}$ is typically needed, while shorter distances are used for measurements in neonates and infants (Koizumi et al.,

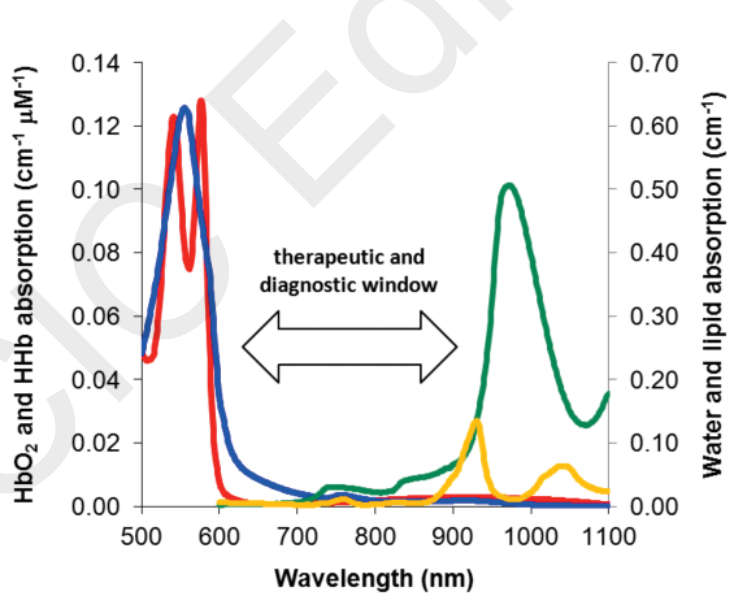

Figure 2 - Absorption spectra of main tissue components in the visible (400-700 nm, VIS) and near infrared $(700-2000 \mathrm{~nm}, \mathrm{NIR})$ spectral regions.

Oxygenated and deoxygenated hemoglobin (red and blue curve, respectively; axis on the left), water and lipid (green and yellow curve, respectively; axis on the right). The so-called therapeutic and diagnostic window is also shown.
2003). Conversely, with TD DOS, the penetration depth is not affected by the source-detector distance, as long as the same photon propagation time $t$ is considered, and a shorter (even null) distance can be used (Torricelli et al., 2005) provided an efficient timegating mechanism is applied to suppress early photons (Pifferi et al., 2008). Due to the combined effect of absorption and scattering on the overall signal attenuation, the greater the distance, the lower the signal-to-noise ratio will be.

The modeling of light propagation in diffusive media is conveniently approached in the framework of the radiative transfer theory where light is treated as a stream of particles (i.e. photons), disregarding its wave nature. From the energy balance (or equivalently the photon balance) in a given volume, it is possible to derive the radiative transfer equation (RTE) which can be solved under the diffusion approximation. Useful closed-form expressions for reflectance and transmittance can be obtained in several simple geometries (e.g. parallelepiped, sphere and cylinder) and even in more complex cases that are more representative of real-life scenarios (e.g. layered medium, inhomogeneity embedded in a homogeneous medium) (Martelli et al., 2010).

\section{Fast optical signal}

The FOS technique has the reputation of being controversial. While several experiments in open-skull animal models have clearly shown the possibility of relating neuronal activation to light scattering changes (Cohen

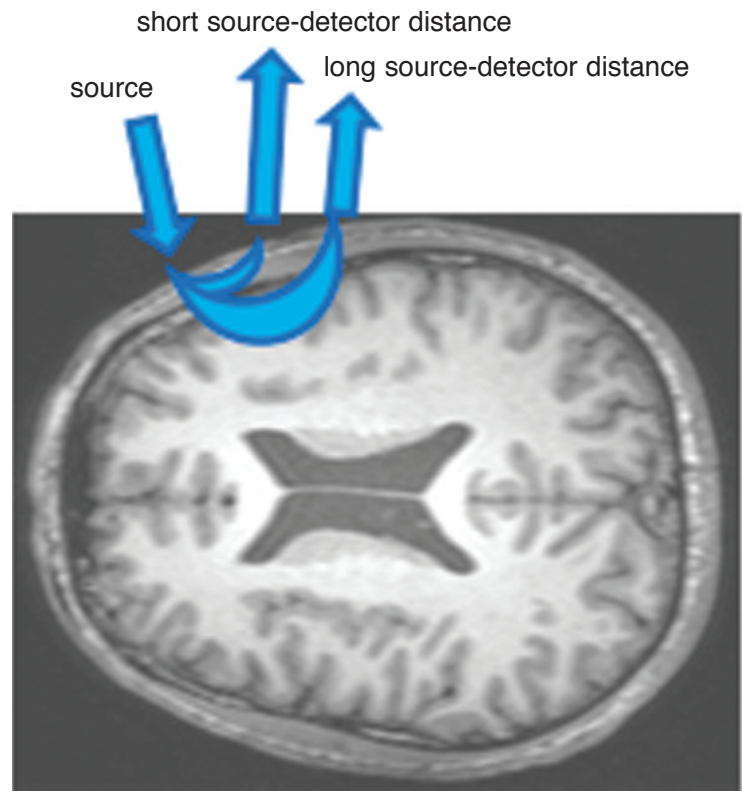

Figure 3 - Scheme of non-invasive CW DOS measurement in reflectance configuration.

A long $(>30 \mathrm{~mm})$ source-detector distance generally allows penetration to cortical surface, while for short $(<10 \mathrm{~mm})$ distances light is confined mostly in extracerebral tissue. A similar scheme for the TD DOS can be found in Torricelli et al. (2014). 
et al., 1972; Frostig et al., 1990; Stepnoski et al., 1991; Rector et al.,1997), application of the technique for non-invasive monitoring of human brain function has given contradictory results. The initial enthusiasm over the FOS technique (Gratton et al., 1998; Rinne et al., 1999; Gratton et al., 2000; Gratton and Fabiani, 2001; Wolf et al., 2002; Franceschini and Boas, 2004; Morren et al., 2004; Gratton et al., 2006; Tse et al., 2006; Parks et al., 2012) has been tempered by negative results (Steinbrink et al., 2000; Syré et al., 2003). A study by Steinbrink et al. (2005) clearly pointed out the critical issues in FOS and suggested that with current technology the signal-to-noise-ratio associated with light intensity changes recorded through the intact head by means of this method is too low to allow the detection of reliable brain cortex activations.

More recently the FOS technique has been revisited (Radhakrishnan et al., 2009; Chiarelli et al., 2012), confirming that its criticality lies in the signal-to-noiseratio. Moreover it was stated that the FOS signal is weak and localized; therefore, it is important not only to correctly use a measurement system with high detection sensitivity, but also to use a properly tailored experimental protocol and to provide a priori anatomical information on the probed area (Sun et al., 2014).

\section{Diffuse correlation spectroscopy}

Diffuse correlation spectroscopy (DCS) is a recent technique that enhances the well-established Laser Doppler flowmetry (LDF) technique. Both DCS and LDF are in fact sensitive to the motion of red blood cells in tissues and record the fluctuations in intensity of coherent light (Bonner and Nossal, 1981). Unlike LDF which has a very limited penetration depth since it uses a very short source-detector distance (e.g. 1 $\mathrm{mm}$ ) (Rajan et al., 2009), DCS employs a sourcedetector distance in the $\mathrm{cm}$ range (e.g. 10-30 mm) and can be used to non-invasively monitor brain cortical activity through the intact scalp and skull in adults (Durduran and Yodh, 2014). Interestingly, LDF measurements performed at long source-detector distance have been reported (Binzoni et al., 2003), but LDF mainly remains associated with short distances and thus with the monitoring of perfusion in superficial tissues (e.g. skin). Furthermore, DCS measures the autocorrelation function of the detected light intensity from which the diffusion coefficient of the moving particle is derived, while LDF typically estimates the spectral power density of light intensity that is related to the autocorrelation function by the Fourier transform theorem.

DCS has recently been revised to account for the long source-detector distances and to present the general physical framework in the context of the diffusion correlation function (Boas et al., 1995).

From the technical point of view, DCS is based on the use of a light source with a coherent length that is longer than the majority of photon path lengths in the tissues that have to be probed. A CW laser with a coherence length of about $10 \mathrm{~m}$ is generally chosen. A single photon detector coupled to a single-mode (or few-mode) optical fiber is used to collect light from the tissue, while a digital hardware correlator is used to compute the autocorrelation function (Durduran et al., 2010; Durduran and Yodh, 2014). While LDF is easily found on the market, to date only one company markets DCS.

DCS has been used in several clinical frameworks and has been extensively validated in vivo against LDF in muscle tissues (Durduran, 2004; Mesquita et al., 2010), color Doppler ultrasound and transcranial Doppler ultrasound in infant and adult brain tissues (Durduran 2004; Yu et al., 2005; Buckley et al., 2009; Menon et al., 2003, Roche-Labarbe et al., 2010; Zirak et al., 2010), phase-encoded velocity mapping MRI in neonatal brain tissue (Buckley et al., 2012), arterialspin labeled MRI in neonatal and adult brain tissues (Durduran, 2004; Yu et al., 2007; Durduran and Yodh, 2014), and Xenon-CT in the injured adult brain (Kim et al., 2010). Overall, these studies suggest that DCS quantitatively determines relative changes in cerebral blood flow quite well with respect to a baseline.

\section{Functional near infrared spectroscopy}

Functional near infrared spectroscopy (fNIRS) measures the absorption changes caused by local variations in cerebral blood volume and oxygenation. The possibility of non-invasively probing hemoglobin in deep biological tissues by monitoring the attenuation of NIR light was first introduced in the late 1970s by Jöbsis (1977) but the application of the technique to the functional study of the brain cortex, by research groups in Europe, Japan and the US, dates back to only 1993 (Hoshi and Tamura, 1993; Villringer et al., 1993). Since then fNIRS has evolved and now it is probably the most mature optical technique for the non-invasive monitoring and imaging of brain function. A historical review on fNIRS can be found in Ferrari and Quaresima (2012), while recently published reviews have focused on the physical and technical issues of CW (Scholkmann et al., 2014) and TD (Torricelli et al., 2014) fNIRS, respectively.

In general an fNIRS instrument can be classified as an oximeter and imager by referring to the possibility to monitor few (maximum 4) distant sites on the head (e.g. left and right frontal and occipital) or to cover a large area of the head so as to create maps of brain cortical activity. The majority of instruments employ the CW modality, possibly modified with the spaceresolved reflectance (SRS) approach, which consists of using multiple detectors in the area surrounding the source position. In this way SRS is able to estimate the photon path in the medium. A CW oximeter typically measures light attenuation changes at a single sourcedetector pair at two or more wavelengths: this enables changes in the tissue concentrations of $\mathrm{HbO}_{2}$ and $\mathrm{Hb}$ to be derived. Conversely, the SRS approach enhances the classical CW approach, given that it is also able to provide an estimate of the so-called tissue oxygenation index, closely related to the blood tissue 
oxygen saturation parameter $\left[\mathrm{SO}_{2}=\mathrm{HbO}_{2} /\left(\mathrm{HbO}_{2}+\mathrm{Hb}\right)\right]$. By using multiple source-detector pairs arranged in an array, topographic optical imagers have been developed (http://fnirs.org/instruments) that allow an approximate lateral localization of the activated area. Recently the high-density approach has been shown to be able to report enhanced spatial resolution as compared to classical imaging approaches (Zeff et al., 2007; Eggebrecht et al., 2014). Noticeably, wearable multichannel systems have recently been introduced and allow the study of brain functions in freely moving subjects (Piper et al., 2014).

In the last decade the availability of several commercial instruments (mainly CW) has fostered a spread of fNIRS within the research community and many endusers (e.g. clinicians) have been using this methodology in fields like brain development, cognitive functions, response to sensorimotor stimuli, psychiatry, neurology and aging. For details see Boas et al., (2014) and the references therein.

\section{Discussion}

Despite the considerable experience accumulated (over a period of more than 20 years), the optical methods for non-invasive brain monitoring herein presented remain confined to a niche in the clinical research arena. There are in fact several critical issues that have hampered their more extensive use in real-life settings: limited spatial resolution, lack of quantification, and impaired reliability.

On the one hand, the diffusive nature of biological tissues in the NIR spectral range is the characteristic that makes non-invasive monitoring of brain function possible, since light can penetrate deeply and eventually be re-emitted at the surface, carrying valuable information on the probed cortical area. On the other hand, light scattering has the negative effect of reducing spatial resolution. While lateral resolution can be improved by employing a dense arrangement of optodes (Zeff et al., 2007; Eggebrecht et al., 2014), as a rule of thumb depth resolution is limited to $5-10 \mathrm{~mm}$, therefore potentially sufficient to discriminate cortical area from overlying extra-cerebral tissues, but definitely not sufficient to finely resolve the hierarchical cortical structure.

The confounding effect of hemodynamic changes occurring in the superficial scalp is now recognized as a major source of error with all optical techniques (Takahashi et al., 2011; Aslin, 2013). The use of TD (Aletti et al., 2012; Kirilina et al., 2012) or SRS (Gagnon et al., 2012) approaches can potentially eliminate or at least reduce this effect. Noticeably these approaches are limited to research prototypes while the majority of commercial devices use a classical CW approach (Ferrari and Quaresima, 2012). Previously, this effect has been found to have a negative impact on use of fNIRS devices in the clinic (Maas and Citerio, 2010).

While accurate estimates of optical properties in simple cases (e.g. homogeneous parallelepiped) are feasible, it should be noted that the human head is geo- metrically complex and optically heterogeneous. A rough model locally approximates the head as a layered structure where scalp, skull, cerebrospinal fluid and gray and white matter have noticeably different optical properties. Analytical models exist to express basic measurable quantities (e.g. reflectance) as a function of source-detector distance, absorption coefficient and reduced scattering coefficients in layered media (Martelli et al., 2010). For a more accurate description, finite element method or Monte Carlo solvers can handle a realistic geometry taken from 3D anatomical MRI or CT scans (http://web4.cs.ucl.ac.uk; http://www.dartmouth.edu/ nir/nirfast; Fang and Boas, 2009). However, the real values of the in vivo optical properties of the different head compartments, and their intra- and inter-subject variations remain poorly determined (Bevilacqua et al., 1999). This reduces the overall matching between simulations and measurements, and may have confounding effects also on the design of tailored instrumentation. Recent studies have been started to provide better estimates of basic optical properties (Farina et al., 2013), but wider research is needed to study the effect of anatomy, aging and physiology on the optical parameter.

The presented optical techniques target different physiological effects linked to brain function, therefore each of them is able to give only a partial picture of the overall phenomenon. Integration of techniques can potentially provide a more complete neurophysiological description. The easiest integration is between DCS and fNIRS since they both address the hemodynamic changes following neuronal activation. The advantage lies in the possibility of simultaneously providing cerebral blood volume and blood flow as well as blood tissue oxygen saturation, and allowing non-invasive estimation of the cerebral metabolic rate of oxygen (Boas et al., 2003). In a few cases, integration of DCS and fNIRS has already been explored at the research level (Roche-Labarbe et al., 2014; Busch et al., 2012; Diop et al., 2011) while a European project (BabyLux, http://www.babylux-project.eu) was recently started to provide the first integrated instrument combining DCS and TD fNIRS for monitoring cerebral oxygen metabolism and blood flow in preterm neonates. Integration of DCS or fNIRS with FOS is fascinating since it would, through an all-optical methodology, provide information on both the fast neuronal changes (usually captured by EEG) and the slow hemodynamic changes (usually measured by PET or fMRI). Today, this is a challenging task that still requires a significant advance at technological and research level.

Integration of optical techniques with other neuroimaging modalities (e.g. EEG, fMRI) is quite straightforward and has been demonstrated by several research groups and also effectively applied in clinical studies (Durduran and Yodh, 2014, Scholkmann et al., 2014, Torricelli et al., 2014).

Finally, a comment on the need for standardization and quality assessment is warranted. There is an increasing awareness in the biophotonic community that this is a key requirement for the translation of neurophotonic tools to the clinics (Hwang et al., 2012). The defini- 
tion of common procedures for the performance assessment of instruments, and the parallel availability of highly calibrated and reproducible phantoms are key requirements for the quantitative assessment of instruments and the validation of clinical prototypes, for quality control and consistency in clinical studies, and for the comparison of clinical results performed with different instruments. Within the framework of different European projects (MEDPHOT, OPTIMAMM, nEUROPt, LaserLabEurope), common protocols and related phantom kits have been developed to provide guidelines for the comparison of various diffuse optical systems (Pifferi et al., 2005; Wabnitz et al., 2011, 2013). Further, a joint initiative of the International Electrotechnical Commission (IEC) and of the International Organization for Standardization (ISO) has been started, aiming at defining a simple, easy-touse standard (http://www.iec.ch). Standardization should also involve data analysis procedures. Within the fNIRS scientific community an initiative for standardization of data types (http://fnirs.org/software) has recently been started, also supported by the availability of open source software (Huppert et al., 2009; Ye et al., 2009). Unfortunately no similar initiatives are, at present, envisaged for DCS and FOS.

\section{Concluding remarks}

The possibility of non-invasively monitoring brain functions by shedding light on the intact head is fascinating and challenging. Given the level of maturity now reached by non-invasive optical techniques like DCS and fNIRS, it is not unrealistic to foresee wider adoption of such modalities in clinical studies. Awareness of the potentiality of these techniques is in fact increasing among end users. On the other hand the FOS technique still necessitates further validation to better identify the optimal working procedures. The advances in photonic technologies in terms of availability of novel and higher-performing components (e.g. light sources and detectors) will stimulate multidisciplinary actions (either at basic or applied research level) aimed at overcoming existing shortcomings. A bright future for neurophotonics lies ahead.

\section{Acknowledgments}

The research leading to these results has partially received funding from BabyLux (grant agreement $n$. 620996, EU CIP ICT PSP).

\section{References}

Aletti F, Re R, Pace V, et al (2012). Deep and surface hemodynamic signal from functional time resolved transcranial near infrared spectroscopy compared to skin flowmotion. Comput Biol Med 42:282-289.

Arridge SR (1999). Optical tomography in medical imaging. Inverse Problems 15:R41-R93.
Aslin RN (2013). Questioning the questions that have been asked about the infant brain using near-infrared spectroscopy. Cogn Neuropsychol 29:7-33.

Ataka K, Pieribone VA (2002). A genetically targetable fluorescent probe of channel gating with rapid kinetics. Biophys $\mathrm{J}$ 82:509-516.

Bevilacqua F, Piguet D, Marquet $P$, et al (1999). In vivo local determination of tissue optical properties: applications to human brain. Appl Opt 38:4939-4950.

Binzoni T, Leung TS, Boggett D, et al (2003). Non-invasive laser Doppler perfusion measurements of large tissue volumes and human skeletal muscle blood RMS velocity. Phys Med Biol 48:2527-2549.

Boas DA, Campbell LE, Yodh AG (1995). Scattering and imaging with diffusing temporal field correlations. Phys Rev Lett 75:1855-1858.

Boas DA, Strangman G, Culver JP, et al (2003). Can the cerebral metabolic rate of oxygen be estimated with nearinfrared spectroscopy? Phys Med Biol 48:2405-2418.

Boas DA, Elwell CE, Ferrari M, et al (2014). Twenty years of functional near-infrared spectroscopy: introduction for the special issue. Neuroimage 85:1-5.

Bohren CF, Huffman DR (1983). Absorption and Scattering of Light by Small Particles. New York, Wiley.

Bonner R, Nossal R (1981). Model for laser Doppler measurements of blood flow in tissue. Appl Opt 20:2097-2107.

Born M, Wolf E (1999). Principles of Optics. Cambridge, Cambridge University Press.

Buckley EM, Cook NM, Durduran T, et al (2009). Cerebral hemodynamics in preterm infants during positional intervention measured with diffuse correlation spectroscopy and transcranial Doppler ultrasound. Opt Express 17:12571-12581.

Buckley EM, Hance D, Pawlowski T, et al (2012). Validation of diffuse correlation spectroscopic measurement of cerebral blood flow using phase-encoded velocity mapping magnetic resonance imaging J Biomed Opt 17:037007.

Busch DR, Choe R, Durduran T et al (2012). Microvascular blood flow changes in human breast during simulated mammography. Biomedical Optics, BIOMED 2012, pp. JM3A.13.

Chiarelli AM, Di Vacri A, Romani GL, et al (2012). Fast optical signal in visual cortex: improving detection by General Linear Convolution Model. Neuroimage 66:194-202.

Cohen LB, Keynes RD, Landowne D (1972). Changes in light scattering that accompany the action potential in squid giant axons: potential-dependent components. J Physiol 224:701-725.

Contini D, Zucchelli L, Spinelli L, et al (2012). Brain and muscle near infrared spectroscopy/imaging techniques. JNIRS Journal of Near Infrared Spectroscopy 20:15-27.

Del Bianco S, Martelli F, Zaccanti G (2002). Penetration depth of light re-emitted by a diffusive medium: theoretical and experimental investigation. Phys Med Biol 47:4131-4144.

Devor A, Boas DA (2012). Neurovascular imaging. Front Neuroenergetics 4:1.

Diop M, Verdecchia K, Lee TY, et al (2011). Calibration of diffuse correlation spectroscopy with a time-resolved nearinfrared technique to yield absolute cerebral blood flow measurements. Biomed Opt Express 2:2068-2081.

Dunn A, Richards-Kortum R (1996). Three-dimensional computation of light scattering from cells. IEEE Journal of Selected Topics in Quantum Electronics 2:898-905.

Durduran T (2004). Non-invasive measurements of tissue hemodynamics with hybrid diffuse optical methods. Ph.D. Dissertation, University of Pennsylvania (http://www. physics.upenn.edu/yodhlab/theses/). 
Durduran T, Choe R, Baker WB, et al (2010). Diffuse optics for tissue monitoring and tomography. Rep Prog Phys 73:076701.

Durduran T, Yodh AG (2014). Diffuse correlation spectroscopy for non-invasive, micro-vascular cerebral blood flow measurement. Neuroimage 85:51-63.

Eggebrecht AT, Ferradal SL, Robichaux-Viehoever A, et al (2014). Mapping distributed brain function and networks with diffuse optical tomography. Nat Photonics 8:448-454.

Fang Q, Boas DA (2009). Monte Carlo simulation of photon migration in $3 \mathrm{D}$ turbid media accelerated by graphics processing units. Opt Express 17:20178-20190.

Farina A, Pifferi A, Torricelli A, et al (2013). Multi-laboratory investigation of the optical properties of the human head. Progress in Biomedical Optics and Imaging - Proceedings of SPIE, 8804 , art. no. 880408.

Ferrari M, Quaresima V (2012). A brief review on the history of human functional near-infrared spectroscopy (fNIRS) development and fields of application. Neuroimage 63:921-935.

Franceschini MA, Boas DA (2004). Noninvasive measurement of neuronal activity with near-infrared optical imaging. Neuroimage 21:372-386.

Frostig RD, Lieke EE, Ts'o DY, et al (1990). Cortical functional architecture and local coupling between neuronal activity and microcirculation revealed by in vivo high-resolution optical imaging of intrinsic signals. Proc Natl Acad Sci U S A 87:6082-6086.

Gagnon L, Cooper RJ, Yücel MA, et al (2012). Short separation channel location impacts the performance of short channel regression in NIRS. Neuroimage 59:2518-2528.

Gibson AP, Austin T, Everdell NL, et al (2006). Three-dimensional whole-head optical tomography of passive motor evoked responses. Neuroimage 30:521-528.

Golovko D, Meier R, Rummeny E, et al (2011). Optical imaging of rheumatoid arthritis. Int J Clin Rheumtol 6: 67-75.

Gratton G, Corballis PM, Cho E, et al (1995). Shades of gray matter: noninvasive optical images of human brain responses during visual stimulation. Psychophysiology 32:505-509.

Gratton G, Fabiani M, Goodman-Wood MR, et al (1998). Memory-driven processing in human medial occipital cortex: an event-related optical signal (EROS) study. Psychophysiology 35:348-351.

Gratton G, Sarno A, Maclin E, et al (2000). Toward noninvasive 3-D imaging of the time course of cortical activity: investigation of the depth of the event-related optical signal. Neuroimage 11:491-504.

Gratton G, Fabiani M (2001). The event-related optical signal: a new tool for studying brain function. Int J Psychophysiol 42:109-121.

Gratton G, Brumback CR, Gordon BA, et al (2006). Effects of measurement method, wavelength, and source-detector distance on the fast optical signal. Neuroimage 32:15761590.

Guerrero G, Siegel MS, Roska B, et al (2002). Tuning FlaSh: redesign of the dynamics, voltage range, and color of the genetically encoded optical sensor of membrane potential. Biophys J 83:3607-3618.

Hill DK, Keynes RD (1949). Opacity changes in stimulated nerve. J Physiol 108:278-281.

Hillman EM (2007). Optical brain imaging in vivo: techniques and applications from animal to man. J Biomed Opt 12:051402.

Hoshi Y, Tamura M (1993). Detection of dynamic changes in cerebral oxygenation coupled to neuronal function during mental work in man. Neurosci Lett 150:5-8.
Huppert TJ, Diamond SG, Franceschini MA, et al (2009). HomER: a review of time-series analysis methods for near-infrared spectroscopy of the brain. Appl Opt 48:D280D298.

Hwang J, Ramella-Roman JC, Nordstrom R (2012). Introduction: Feature issue on phantoms for the performance evaluation and validation of optical medical imaging devices. Biomed Opt Express 3:1399-1403.

Jacques SL (2013). Optical properties of biological tissues: a review. Phys Med Biol 58:R37- R61.

Jöbsis FF (1977). Noninvasive, infrared monitoring of cerebral and myocardial oxygen sufficiency and circulatory parameters. Science 198:1264-1267.

Kim MN, Durduran T, Frangos S, et al (2010). Noninvasive measurement of cerebral blood flow and blood oxygenation using near-infrared and diffuse correlation spectroscopies in critically brain-injured adults. Neurocrit Care 12:173-180.

Kirilina E, Jelzow A, Heine A, et al (2012). The physiological origin of task-evoked systemic artefacts in functional near infrared spectroscopy. Neuroimage. 61:70-81.

Koizumi H, Yamamoto T, Maki A, et al. (2003). Optical topography: practical problems and new applications. Appl Opt 42:3054-3062.

Lipton P (1973). Effects of membrane depolarization on light scattering by cerebral cortical slices. J Physiol 231:365-383.

Maas Al, Citerio G (2010). Noninvasive monitoring of cerebral oxygenation in traumatic brain injury: a mix of doubts and hope. Intensive Care Med 36:1283-1285.

MacVicar BA, Hochman D (1991). Imaging of synaptically evoked intrinsic optical signals in hippocampal slices. $J$ Neurosci 11:1458-1469.

Malonek D, Grinvald A (1996). Interactions between electrical activity and cortical microcirculation revealed by imaging spectroscopy: implications for functional brain mapping. Science 272:551-554.

Martelli F, Del Bianco S, Ismaelli A, et al (2010). Light Propagation through Biological Tissue and Other Diffusive Media: Theory, Solutions, and Software. Washington, USA, SPIE Press.

Menon C, Polin GM, Prabakaran I, et al (2003). An integrated approach to measuring tumor oxygen status using human melanoma xenografts as a model. Cancer Res 63:72327240.

Mesquita RC, Skuli N, Kim MN, et al (2010). Hemodynamic and metabolic diffuse optical monitoring in a mouse model of hindlimb ischemia. Biomed Opt Express. 1:1173-1187.

Morren G, Wolf U, Lemmerling P, et al (2004). Detection of fast neuronal signals in the motor cortex from functional near infrared spectroscopy measurements using independent component analysis. Med Biol Eng Comput 42:92-99.

Mourant JR, Freyer JP, Hielscher AH, et al (1998). Mechanisms of light scattering from biological cells relevant to noninvasive optical-tissue diagnostics. Appl Opt 37:3586-3593.

Nieuwenhuizen RP, Lidke KA, Bates M, et al (2013) Measuring image resolution in optical nanoscopy. Nat Methods 10 : 557-562.

Obrig $\mathrm{H}$, Villringer A (2003). Beyond the visible-imaging the human brain with light. J Cereb Blood Flow Metab 23:1-18.

Ogawa S, Tank DW, Menon R, et al (1992). Intrinsic signal changes accompanying sensory stimulation: functional brain mapping with magnetic resonance imaging. Proc Natl Acad Sci USA 89:5951-5955.

Parks NA, Maclin EL, Low KA, et al (2012). Examining cortical dynamics and connectivity with simultaneous single pulse transcranial magnetic stimulation and fast optical imaging. Neuroimage 59:2504-2510. 
Pifferi A, Torricelli A, Bassi A, et al (2005). Performance assessment of photon migration instruments: the MEDPHOT protocol. Appl Opt 44, 2104-2114.

Pifferi A, Torricelli A, Spinelli L, et al (2008). Time-resolved diffuse reflectance using small source-detector separation and fast single-photon gating. Phys Rev Lett 100:138101.

Piper SK, Krueger A, Koch SP, et al (2014). A wearable multichannel fNIRS system for brain imaging in freely moving subjects. Neuroimage 85:64-71.

Popp J, Tuchin VV, Chiou A, Heinemann S (Eds) (1999). Handbook of Biophotonics. New York, John Wiley and Sons.

Radhakrishnan H, Vanduffel W, Deng HP, et al (2009). Fast optical signal not detected in awake behaving monkeys. Neuroimage 45:410-419.

Rajan V, Varghese B, van Leeuwen TG, et al (2009). Review of methodological developments in laser Doppler flowmetry. Lasers Med Sci 24:269-283.

Rector DM, Poe GR, Kristensen MP, et al (1997). Light scattering changes follow evoked potentials from hippocampal Schaeffer collateral stimulation. J Neurophysiol 78:17071713.

Rinne T, Gratton G, Fabiani M, et al (1999). Scalp-recorded optical signals make sound processing in the auditory cortex visible? Neuroimage 10:620-624.

Roche-Labarbe N, Carp SA, Surova A, et al (2010). Noninvasive optical measures of CBV, StO(2), CBF index, and $\mathrm{rCMRO}(2)$ in human premature neonates' brains in the first six weeks of life. Hum Brain Mapp 31: 341-352.

Roche-Labarbe N, Fenoglio A, Radhakrishnan H, et al (2014). Somatosensory evoked changes in cerebral oxygen consumption measured non-invasively in premature neonates. Neuroimage 85:279-286.

Salzberg BM, Obaid AL (1988). Optical studies of the secretory event at vertebrate nerve terminals. J Exp Biol 139:195231.

Scholkmann F, Kleiser S, Metz AJ, et al (2014). A review on continuous wave functional near-infrared spectroscopy and imaging instrumentation and methodology. Neuroimage 85:6-27.

Siegel MS, Isacoff EY (1997). A genetically encoded optical probe of membrane voltage. Neuron 19:735-741.

Steinbrink J, Kohl M, Obrig H, et al (2000). Somatosensory evoked fast optical intensity changes detected non-invasively in the adult human head. Neurosci Lett 291:105108.

Steinbrink J, Kempf FC, Villringer A, et al (2005). The fast optical signal - robust or elusive when non-invasively measured in the human adult? Neuroimage 26:996-1008.

Stepnoski RA, LaPorta A, Raccuia-Behling F, et al (1991). Noninvasive detection of changes in membrane potential in cultured neurons by light scattering. Proc Natl Acad Sci U S A 88:9382-9386.

Sun B, Zhang L, Gong H, et al (2014). Detection of optical neuronal signals in the visual cortex using continuous wave near-infrared spectroscopy, Neuroimage 87:190-198.

Syré F, Obrig H, Steinbrink J, et al (2003). Are VEP correlated fast optical changes detectable in the adult by non-invasive near infrared spectroscopy (NIRS)? Adv Exp Med Biol 530:421-431.
Takahashi T, Takikawa Y, Kawagoe R, et al (2011). Influence of skin blood flow on near-infrared spectroscopy signals measured on the forehead during a verbal fluency task. Neuroimage 57:991-1002.

Taroni P, Pifferi A, Quarto G, et al (2012). Diffuse optical imaging: application to breast imaging (Chapter 9). In: Anastasio A, La Riviere P (Eds) Emerging Imaging Technologies in Medicine. Boca Raton, Florida, CRC Press.

Torricelli A, Pifferi A, Spinelli L, et al (2005). Time-resolved reflectance at null source-detector separation: improving contrast and resolution in diffuse optical imaging. Phys Rev Lett 95:078101.

Torricelli A, Contini D, Pifferi A, et al (2014). Time domain functional NIRS imaging for human brain mapping. Neuroimage 85:28-50.

Tse CY, Tien KR, Penney TB (2006). Event-related optical imaging reveals the temporal dynamics of right temporal and frontal cortex activation in pre-attentive change detection. Neuroimage 29:314-320.

Villringer A, Chance B (1997). Non-invasive optical spectroscopy and imaging of human brain function. Trends Neurosci 20:435-442.

Villringer A, Planck J, Hock C, et al (1993). Near infrared spectroscopy (NIRS): a new tool to study hemodynamic changes during activation of brain function in human adults. Neurosci Lett 154:101-104.

Wabnitz H, Pifferi A, Torricelli A, et al (2011). Assessment of basic instrumental performance of time-domain optical brain imagers. Progress in Biomedical Optics and Imaging - Proceedings of SPIE, 7896, art. no. 789602.

Wabnitz H, Jelzow A, Mazurenka M, et al (2013). Performance assessment of time-domain optical brain imagers: A multilaboratory study. Progress in Biomedical Optics and Imaging - Proceedings of SPIE, 8583, art. no. $85830 \mathrm{~L}$

Welberg L (2013) Techniques: optogenetics takes more control. Nat Rev Neurosci 14:587.

Wolf M, Wolf U, Choi JH, et al (2002). Functional frequencydomain near-infrared spectroscopy detects fast neuronal signal in the motor cortex. Neuroimage 17:1868-1875.

Ye JC, Tak S, Jang KE, et al (2009). NIRS-SPM: statistical parametric mapping for near-infrared spectroscopy. Neuroimage 44:428-447.

Yu G, Durduran T, Zhou C, et al (2005). Noninvasive monitoring of murine tumor blood flow during and after photodynamic therapy provides early assessment of therapeutic efficacy. Clin Cancer Res 11: 3543-3552.

Yu G, Floyd T, Durduran TF, et al. (2007). Validation of diffuse correlation spectroscopy for muscle blood flow with concurrent arterial spin labeled perfusion MRI. Opt Express 15:1064-1075.

Zeff BW, White BR, Dehghani H, et al (2007). Retinotopic mapping of adult human visual cortex with high-density diffuse optical tomography. Proc Natl Acad Sci U S A 104:12169174.

Zhu D, Larin KV, Luo Q, et al (2013). Recent progress in tissue optical clearing. Laser Photon Rev 7:732-757.

Zirak P, Delgado-Mederos R, Martí-Fàbregas J, et al (2010). Effects of acetazolamide on the micro- and macro-vascular cerebral hemodynamics: a diffuse optical and transcranial doppler ultrasound study. Biomed Opt Express 1:1443-1459. 